\title{
Evaluation of Phlebotomy Services in Clinical Laboratory Setting in Addis Ababa Public Hospitals, Addis Ababa, Ethiopia
}

\author{
Wondimeneh Liknaw Mekonon ${ }^{1}$, Aster Tsegaye Abebe ${ }^{2}$, Eshetu Lemma Haile ${ }^{3}$, \\ Abay Sisay Misganaw, ${ }^{2, *}$ \\ ${ }^{1}$ ALERT Center, Addis Ababa, Ethiopia \\ ${ }^{2}$ School of Clinical Laboratory Science, College of Health Science, Addis Ababa University, Addis Ababa, Ethiopia \\ ${ }^{3}$ GIZ-Ethiopia, Addis Ababa, Ethiopia
}

Email address:

abusis27@gmail.com (A. S. Misganaw),wondimenehliknaw@gmail.com (A. S. Misganaw)

*Corresponding author

\section{To cite this article:}

Wondimeneh Liknaw Mekonon, Aster Tsegaye Abebe, Eshetu Lemma Haile, Abay Sisay Misganaw. Evaluation of Phlebotomy Services in Clinical Laboratory Setting in Addis Ababa Public Hospitals, Addis Ababa, Ethiopia. American Journal of Laboratory Medicine.

Vol. 2, No. 3, 2017, pp. 24-33. doi: 10.11648/j.ajlm.20170203.11

Received: April 18, 2017; Accepted: April 28, 2017; Published: June 23, 2017

\begin{abstract}
Background: Phlebotomy is the system of drawing a blood sample for the use of laboratory testing and for blood transfusion. Professionals who are performing phlebotomy services called phlebotomists. It is a critical part of the pre analytical phase of laboratory testing and is the most neglected procedures in health care. About $70 \%$ of the quality of the test are affected during phlebotomy and other pre analytical services. However, little is known about the practice of phlebotomy services in developing countries like Ethiopia. Objectives: To assess the practice of phlebotomists and to identify the major sources of errors during venous blood collection in public hospitals in Addis Ababa. Methodology: Hospital based, cross sectional observational and follow-up study was conducted from March to May 30, 2014. The study followed 40 phlebotomists while each of them was collecting 5 different venous blood collections (giving a total of 200 phlebotomies). Well structured questionnaires and checklists were used to collect data. Data was entered on EPI-Data version 3.1 and statistical analysis was performed with SPSS version 20. Descriptive statistics were employed and the Chi square test was used for comparing major errors observed. Result: Almost all laboratory phlebotomy sites had no Standard Oprating Procedures (SOPs) available in collection sites and most of collection sites were not well ventilated. The major errors identified were use of single glove for more than one client 139 of 200 (69.5\%), inappropriate cleaning practice of vein puncture sites 180 of $200(90 \%)$, collecting blood before the disinfectant alcohol dried 139 of 200 (69.5\%), incorrect tube collection sequences 107 of $200(53.5 \%$ ), unnecessarily applying of tourniquets after blood started flowing in to the collection tubes and syringes 170 of $200(85.0 \%)$ and applying tourniquets before locating and selecting appropriate site for venous blood collection 175 of 200 (87.5\%). Conclusion and Recommendation: -Many errors were identified in the phlebotomy practice during the observational study. As the quality of blood specimen influences patient result: emphasis should be given on phlebotomy training to improve the practices for phlebotomists and ensure safety as well as quality during blood collections for laboratory analysis.
\end{abstract}

Keywords: Phlebotomy, Venous Blood Collection, Quality, Tourniquet, Preanalytical Errors

\section{Introduction}

Phlebotomy is the system of drawing a blood sample for the use of laboratory testing and for blood transfusion. Professionals who are performing phlebotomy services called phlebotomists and those who are undertaking phlebotomy need to be trained in procedures specific to the types of services they will perform [1]. During the late 1980s and early 1990s, the phlebotomy profession emerged as a result of technology and expansion of laboratory roles. Primarily, only medical technicians and medical technologists were responsible for collecting blood samples, but as technology 
and the health care industry remarkably advanced in technology, particularly during the last two decades by other health professionals and trained phlebotomists share these responsibilities [2].

In recent years, the interest of the clinical laboratory has been increasing towards quality improvement and patient safety activities in healthcare [3]. Clinical laboratories are now conforming to certification rules of the International Organization for Standardization (ISO) for accreditation programs with standards based on ISO 15189 [4, 5]. While the laboratory quality testing process involves three phases: pre-analytical, analytical and post analytical phases'. A major problem in laboratory quality management is that health care professionals still focus excessive attention on the analytical phase of laboratory testing. Accreditation agencies are increasingly requiring laboratories to go beyond the analytical quality and take responsibility for pre- and postanalytical where most errors arise $[3,4,6]$.

About $46-68.2 \%$ of total laboratory errors occur in the preanalytic phase $[7,8]$. Of those errors, most errors are occurd during blood sample collection [9].

Among factors influencing the outcome of laboratory results during blood collection could be due to problems on phlebotomist education and training, including understanding of anatomy, prevention and control of safety issues during blood collection, incorrect use of the recommended blood collection sequence of vacuum tubes and the volume to be filled, problems on maintaining good condition of the sample and safe transportation of the blood sample are among the most common errors identified [10].

The pre-analytical phase, especially venous blood collection is most critical in influencing patients laboratory results derived from blood specimens. Because phlebotomy is the most regularly practiced but, neglected medical process worldwide. It can lead to adverse problems in patient safety and healthy life if not properly regulated. Patient identification problem, usage of incorrect blood collection equipment and improper skin puncturing practices are most problems occurred during phlebotomy service and also laboratory results can be affected during tourniquet application, improper usage of disinfectants [11], improperly using of Vacutainer tube sequence during collection and improper patient identification and instruction before collection [12].

In order to have clear view of shortcomings, knowledge gaps on the laboratory phlebotomy practice and the challenges about laboratory phlebotomy services need to be assessed. Studies on evaluation of phlebotomy services in clinical laboratory setting and the factors which influence quality of clinical specimens in Ethiopia have not been documented, as far as our knowlege goes. Therefore, this study was carried out to evaluate phlebotomy services in the clinical laboratory and factors affecting the quality of venous blood specimens in Addis Ababa public hospitals. Assessing the status and identifying gaps will help design appropriate intervention.

\section{Methodology}

\subsection{Study Area}

The study was conducted in selected public health facilities in Addis Ababa which is the largest city in Ethiopia. The city has 46 hospitals, 11 are public hospitals, of which 6 are under Addis Ababa Regional Health Bureau (AARHB) and 5 are specialized referral ones (one University Hospital and 4 of them under the Federal Ministry of Health). Three are military hospitals, 4 are NGO's and the rest 28 are private hospitals [13].

This study focused on 11 public hospitals in the city and surrounding areas which use a Vacutainer tube for blood collection. Those were Tikur Anbessa Specialized teaching hospital, St. Peters TB specialized hospital, ALERT center, St. Paul's Hospital Millenium Medical college, Zewditu Memorial Hospital, Yekatit 12 hospitals, Minilik II hospital, Ras Desta Damtew hospital, Tirunesh Beijing hospital, Emanuel hospital and Ghandi memorial hospitals. One of the hospitals (Emanuel Hospital) selected for piloting by using a lottery selection method.

\subsection{Study Design}

Hospital based, observational and interview based cross sectional type study was conducted between March to May 30, 2014. In this study both quantitative and qualitative design, data collection method were used. Observational follow up study was performed that the practice of phlebotomists were observed while they collect the blood specimen. Phlebotomists were considered for interview about their practice towards phlebotomy services using a structured questionnaire. Patients who came for phlebotomy services were interviewed about their demographic and voluntarily participation based on the inclusion criteria. Observational checklist was used to assess the practice of phlebotomists using recommended technique. The performance of each Phlebotomist was monitored in 5 different phlebotomies (blood collection practices) and each activity performed during the blood collection time was collected and recorded.

\subsection{Study Period}

Pre testing: Data for pre-test were collected before two weeks of actual data collection started.

Actual data collection time: actual data collection was conducted from March-May 30, 2014.

Source population

The source populations were all phlebotomists who were performing blood collection on out patients in public hospitals, Addis Ababa.

\subsection{Study Population}

All phlebotomists in the selected governmental hospitals in Addis Ababa who were performing venous blood collection using Vacutainer tube system were our study population.

Inclusion Criteria

All voluntary phlebotomists and patients were included 
and patients who came with at least two different type of venous blood collection requests (like for hematology, PT/PTT, chemistry and more others) were included.

\subsection{Study Sample}

The required sample size was determined by using single proportion formula considering the following assumptions:

Negative prevalence $(1-\mathrm{p})=50 \%$

Phlebotomist performance; $50 \%$ was taken due to the absence of reliable previous study that show the performance of Phlebotomist, Level of significance $(\mathrm{p}-$ value $)=0.05$ (5\%), Marginal of error (d) $=0.15(15 \%)$ was used to minimize sample size, Non-response rate $=10 \%$

The formula for calculating the sample size (n) was $\mathrm{n}=\frac{(Z \alpha / 2)^{2} \times P(1-P)}{d^{2}}$

Where: $\mathrm{n}=$ total sample size, $\mathrm{n}_{\mathrm{i}}$ - Sample size calculated from infinite population, $\mathrm{Z}(\alpha / 2)=\mathrm{Z}$-score at $95 \%$ confidence interval $=1.96, \mathrm{P}=$ positive prevalence (assuming that $50 \%$ of the phlebotomist were having good performance) $(1 / 2=0.5)$

$1-\mathrm{P}=$ negative prevalence/proportion $(1 / 2=0.5), \mathrm{d}=$ marginal error $0.15(15 \%)$ is used to minimize sample size. $\Rightarrow$ $\mathrm{n}_{\mathrm{i}}=(1.96)^{2} * 0.5(1-0.5)$

$(0.15)^{2}$

$\Rightarrow \mathrm{n}_{\mathrm{i}}=42.6=43$ 4.3

$\Rightarrow$ By adding $10 \%$ non-response rate to $\mathrm{n}_{\mathrm{i}}=43 \times 10 \% \Rightarrow$

$\Rightarrow$ So total sample size $=\mathrm{n}_{\mathrm{i}}+10 \%$ non-response rate

$\Rightarrow \mathrm{n}=43+4.3=47$ phlebotomists were included from 11 public hospitals, the Equal proportion of phlebotomists; here there are 11 hospitals, 47 phlebotomists

$\Rightarrow 47 / 11$

$\Rightarrow 4.2 \Rightarrow 4$ phlebotomists from each hospital were supposed to be included

The study followed phlebotomists when each phlebotomist is collecting 5 different venous blood collections, so the total of 235 (47 x 5) venous blood collections were included.

\subsection{Sampling Procedures}

All 11 public hospitals laboratories in the Addis Ababa city were included. The total sample size was 47 phlebotomists who were found during the study period and each of them was evaluated in five different phlebotomies. Patients were selected by a convenient sampling method as long as the inclusion criteria is fulfilled, after each follow up and observational study for each patient was finished. In addition, to prevent bias and alerting of the Phlebotomist of their actual practice, each Phlebotomist was interviewed after all follow up and observational study of all Phlebotomist practices were finished.

Data collection method

Structured questionnaires and checklists were used for the interview and observational or follow up techniques. The contents of the questionnaire included socio demographic characteristics, education, training, work experience of Phlebotomist and the practices during phlebotomy services were assessed.

A number of questions that could address the objective of this study were gathered and adapted. In order to improve the developer questionnaire, valuable comments were incorporated from different sources. The first draft questionnaire was English version and then translated to Amharic Language. The layout of the questionnaire was designed to be easy to read and number of pages were limited, to ensure that the questionnaire could be completed within a reasonable period of time. It was pretested for the contents and reliability in phlebotomy context, then correction was done accordingly. The data were collected from the study hospitals by experienced medical laboratory professionals and training were given to data collectors and supervisors about the collection of data until they all well understood the aim and method of collection. Factors that were looked during evaluation of the Phlebotomist performance were:

a. Time of tourniquet application

b. Proper identification of requests to patients before and after blood draw to check their experience of identification and way of communication with patients

c. Excessively aggressive disinfection of the forearm by the phlebotomist, which can produce venous stasis

d. The order in which vacuum tubes were used during specimen collection, Proper labeling and adequacy of mixing of blood in primary vacuum tubes that contain anticoagulant or clot-activating additives and other relevant information.

e. If the phlebotomists become informed about the study, it might alert for the phlebotomies and might take special concern during blood collection which would bias the study. To minimize the bias the laboratory managers were the only personnel who were informed about this research; the phlebotomists were unaware why the data are being collected. The performances of the phlebotomists were evaluated only during procedures that involve blood collection in vacuum tubes (i.e. Coagulation activator, sodium citrate, ethylene diaminetetra acetic acid [EDTA], heparin, or sodium fluoride). The performance of each phlebotomist was monitored in 5 different collections and after the observation finished phlebotomist were questioned about their practice.

\subsection{Study Variables}

\subsubsection{Dependent Variables}

Measure phlebotomist veins blood collection practices in the laboratory. That is,

a. Proper identification of the right patients before collection,

b. Tourniquet application time during collection,

c. Cleaning of puncturing sites,

d. Order of tube during collection,

e. Mixing of collecting samples,

f. Labeling 


\subsubsection{Independent Variables}
a. Gender
b. Education
c. Phlebotomy training

\subsection{Data Processing and Analysis}

Data entry and statistical analysis were performed using EPI-Data version 3.1, SPSS version 20 (Statistical Package for the Social Sciences). Descriptive statistical analysis were used such as percentage, number and data were presented as tables and figures. Major source of errors was compared by using chi square tests. Descriptive statistics and chi square tests were analyzed with SPSS software, version 20. P-values less than 0.05 were considered as statistically significant.

\subsection{Data Quality Assurance}

The questionnaire was pre-tested two weeks before the actual data collection. One day training was given to the data collector, supervisor and a questionnaire were also prepared by local language to facilitate data collection. Data collectors were instructed to check the completeness of each questionnaire at the end of each interview. The supervisor rechecked the completeness of the questionnaire immediately after an interview at the spot. The principal investigator also checked the completeness of the data immediately when the data was submitted.

\subsection{Ethical Considerations}

Ethical clearance was obtained from Departmental Research and Ethics Review Committee (DRERC) of Department of Medical Laboratory Sciences, College of Health Science, Addis Abba University, Addis Ababa Health Bureau Research Ethical committee, ALERT/AHRI ethical committee, St Peter TB specialized hospital ethical clearance committee and St Paul's Hospital Millenium Medical college ethical committee. To maintain confidentiality, data collectors asked patients' and phlebotomists to participate in the study before the interview. Then those participants willing to participate were included in the study. Data collectors informed respondents that they have full right to discontinue or refuse to participate in the study at any time. A letter of agreement was attached to the questionnaire to obtain the written consent of each individual.

\subsection{Operational Definition}

Additive - in a specimen collection tube, any ingredient that is placed in a collection container to facilitate an intended function (e.g. to prevent the blood from clotting or to prevent glycolysis)

Appropriate cleaning: is cleaning of puncture site should be cleaned by starting from the centre of the venipuncture site and work outwards by covering an area of $2 \mathrm{~cm}$ diameter slowly

Evacuate: create a vacuum in or removing of air from the tube

Good labeling: labeling of blood collection test tubes with unique ID, patient name/ initial, collection date and time, collectors name/initial, address of requesters' and requester name/ initial and signature

Patient preparation: Identifying and checking of state of condition of patients before blood collection that will affect / mislead quality of test results

Phlebotomy: is the act or practice of opening a vein by incision or puncture by needle to collect blood.

Phlebotomist: A person who performs phlebotomy has the title "Phlebotomist", and can be laboratory professionals, doctors, nurses or trained persons.

Performances: ability to do the assigned works

Process: the activities that constitute the patient-provider interaction, including diagnosis, treatment and prevention activities etc.

Quality: doing the right thing in the right way at the right time for the right patients.

Tourniquet: In regards to Venipuncture a constrictive band placed over an extremity to distend the veins for the purpose of blood aspiration or intravenous injections. The Materials used may be rubber, latex, or other synthetic elastic material. A blood pressure cuff may also be used.

\section{Results}

\section{General information}

A total of 40 phlebotomists and 200 patients from ten public hospitals of Addis Ababa were involved in the study. Piloting was done at Emanuel hospital Hospital, which one of the hospitals found in AA, and selected by using the lottery method. Phlebotomists were evaluated when they were collecting venous blood samples from five different clients who were coming from getting laboratory service.

Baseline information about the hospital phlebotomy area

The study assessed the phlebotomy area of each hospital. 9 of the 10 hospitals have clear sign of direction. Eighty percent ( 8 of 10) of the phlebotomy area had clean waiting area and in $70 \%$ (7 of 10$)$ had patients waiting area outside of the blood collection area. Seventy percent ( 7 of 10) of the blood collection area had sufficient space to precede their works and had a separate cleaning area but 50\% (5 of 10) of the phlebotomy areas had no clean water, access to keep hygienic. $90 \%$ (9 of 10) of them had cleanable surface and blood drawing table but $70 \%$ (7 of 10) of phlebotomy areas were not ventilated. Phlebotomy areas were not appropriate to secure patients' confidentiality as other patients were around when patients are giving blood specimen in 30\% (3 of 10) of phlebotomy sites Ninety percent (9 of 10) of phlebotomy sites had no any SOPs found during the study time but $50 \%$ ( 5 of 10 ) of them posted job aids which directs how to collect and process blood and other specimens.

\section{Socio Demographic Characteristics}

Out of the 40 phlebotomist 25(62.5\%) were females and $15(37.5 \%)$ were males and of the 200 participated clients on whom phlebotomists practice was followed, 107(53.5\%) were females and 93(46.5\%) were males. The average $( \pm$ SD) age of patients were $31.68( \pm 17.54)$ years (ranges from 2-74 
years), whereas the mean $( \pm \mathrm{SD})$ age of phlebotomists were $31.65( \pm 9.7)$ and they were found in the age group of 20-29 $(52.5 \%), 30-39(25 \%), 40-49(15 \%)$ and $50-59(7.5 \%)$ (Figure 1). Nineteen (47.5\%) of the phlebotomist had monthly income within range of 1001-1500. Twenty-one $(52.5 \%), 8(20 \%), 6(15.0 \%)$, and $12.5 \%$ (5 of 40$)$ of the phlebotomists were educated diploma in laboratory, degree and above, certificate, and $\leq 12$ grade, respectively.

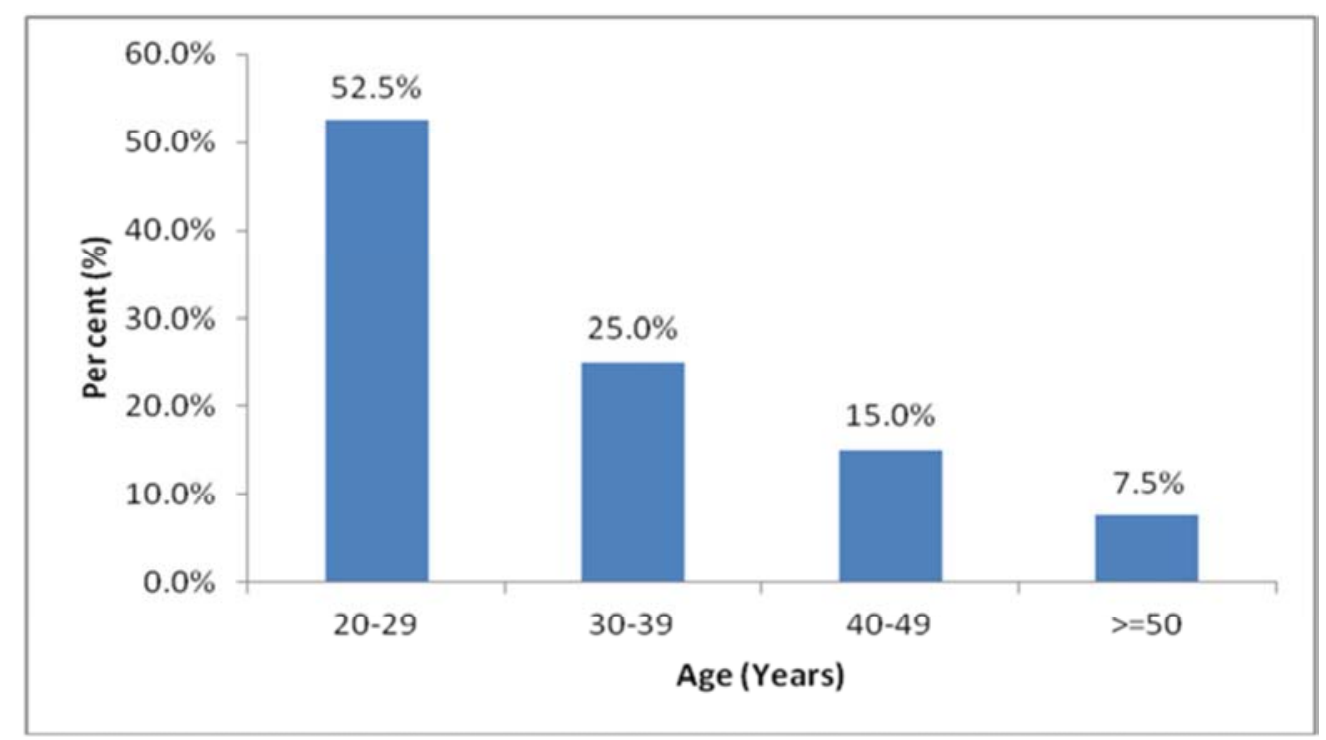

Figure 1. Age (in Years) distributions of phlebotomists in public hospitals in A A, May 2014.

Knowledge of the phlebotomist about general phlebotomy service

About 55\% (22 of 40) phlebotomists had no idea about the recommended tourniquet application time whereas only $37.5 \%$ (15 of 40 ) knew too long application would have impacts on laboratory test results. The Majority of them $60 \%$ (24 of 40) of phlebotomist said they were using collection sequences as Ethylenediaminetetraacetic acid (EDTA) tube firstly and Serum separator tube (SST)/ plane (dry tube) second. While $85 \%$ (34 of 40 ) of them were thinking that SST tube with additive does not need mixing, $40 \%$ (16 of 40) said collected blood with EDTA tube were mixed or inverted $1-4 x$ times. Moreover, 92.5\% (37 of 40) of phlebotomist reported that they are using the recommended elastic tourniquets during phlebotomy.

All (100\%) Phlebotomists agreed that recapping of needles is performed by using one hand principle and disposed used sharps with sharp containers. Regarding hand hygiene, 70\% ( 28 of 40) of phlebotomist said that they have performed hand hygiene before and after blood collection and after removal of gloves. While $75 \%$ (30 of 40) of phlebotomists said they do not know for how many minutes to let the tourniquet applied during venous blood collection, $62.5 \%$ ( 25 of 40 ) even did not know the impacts of applying tourniquets too long on laboratory results. But $67.5 \%$ (33 of 40 ) of them agreed as they have general knowledge about phlebotomy service. Regarding labeling, $57.5 \%$ ( 23 of 40 ) of phlebotomists reported that they always label the time of blood collection, however, only $3(7.5 \%)$ reported correct time for labeling (that is after collection and before the patient leaves).

Observational findings on patient preparation and handling during venous blood collection

Forty phlebotomists were followed while each of them performs 5 phlebotomy procedures on a total of 200 patients during observational study. Accordingly, phlebotomists were not easily identified for 108 of 200 (54\%) of patients and 126 of $200(63 \%)$ of patients were not asked any permission from phlebotomists before blood collection. Phlebotomists did not change new gloves for 134 of $200(67 \%)$ of patients. They apply tourniquets without observing the presence of good vein visually for 175 of $200(87.5 \%)$ of blood collection procedures; they just ask patients to extend their arms, apply a tourniquet and then start to locate the vein. (Table 1).

Table 1. Observational findings on patient preparation and handling during venous blood collection in public hospitals in Addis Ababa, May 2014.

\begin{tabular}{lll}
\hline Procedures & Frequency & Percent \\
\hline $\begin{array}{l}\text { Phlebotomist introducing/ easily identifiable for } \\
\text { patients }\end{array}$ & & \\
Yes & 92 & 46.0 \\
No & 108 & 54.0 \\
Ask minor permission before collecting & & \\
Yes & 74 & 37.0 \\
No & 126 & 63.0 \\
Phlebotomist wear new gloves for each patient & & \\
Not wear glove & 5 & 2.5 \\
Yes & 61 & 30.5 \\
No & 134 & 67.0 \\
Cleaning blood collection site with 70\% & & \\
alcohol swabs & & \\
Yes & 195 & 97.5 \\
No & 5 & 2.5 \\
Cleaning site with gentle pressure & & \\
Not clean at all & 5 & 2.5 \\
Yes & 15 & 7.5 \\
No & 180 & 90.0 \\
Collecting blood after cleaning alcohol dried & & \\
from the blood collection site & & \\
Yes & 61 & 30.5 \\
No & 139 & 69.5 \\
\hline
\end{tabular}




\begin{tabular}{lll}
\hline Procedures & Frequency & Percent \\
\hline $\begin{array}{l}\text { Inappropriate requesting to clenching fist } \\
\text { during collection }\end{array}$ & \\
Yes & 101 & 50.5 \\
No & 99 & 49.5 \\
Touching of the clean site & & \\
Yes & 66 & 33.0 \\
No & 134 & 67.0 \\
Repeat cleaning of touched cleaned collection & & \\
site & & \\
Yes & 28 & 14.0 \\
No & 38 & 19.0 \\
Not applicable & 134 & 67.0 \\
\hline
\end{tabular}

Observation Finding during venous blood collection

Only $35 \%$ of the phlebotomists use vacutainer tubes with vacutainer needle to collect blood sample, while most of the used syringe and transfer blood to vacationer tube; $60.5 \%$ (121) of blood collected were transferred by removing the needle from syringes and by opening vacuum tube. Regarding the sequence of blood draw, 53.5\% (107 of 200) of the phlebotomies were performed following unrecompensed order of tube collection sequences. With the collected blood with additive, $60.5 \%$ were mixed gently to prevent hemolysis. During $85 \%$ (170) of the collections, tourniquets were not released after blood starts to flow into the syringes and tubes. Labeling of test tubes was performed before blood collection and before checking of the patients in $51 \%$ (102) of blood collections.

\section{Observational finding after venous blood collection}

All of the phlebotomists were applying cottons in collecting sites that were used for cleaning skin site for blood collection and when they were asked why they use these dirty swabs for stopping blood, most of them have no reason. Phlebotomists were recapping needles in about $89 \%$ of collections by using one hand principle which was very helpful to minimize needle stick injury; for that reason most of the phlebotomists said that they have never exposed to needle stick injury. Most phlebotomists were putting blood samples on test tube rack immediately after blood collections, but $78.5 \%$ (157) of the collections were not mixed or unacceptable mixing time was practiced after collection (Table 2). Of note, during the study time hand hygiene was not performed in all the studied phlebotomy services by phlebotomists.

A statistically significant finding was not observed in using new gloves for new patients $(\mathrm{p}=0.00)$, wrong tourniquet application after blood flow into the tube or syringes $(\mathrm{p}=0.000)$, incorrect tube collection sequences $(\mathrm{p}=0.00)$ and wrongly applying tourniquets before locating a good vein $(p=0.007)$. In all cases, those below diploma level perform better, as summarizes in table 3 common source of errors observed during phlebotomies based on phlebotomy training distributions (trained $=16$ and untrained $=24$ ). As shown in table 3, statistically significant differences were observed on not using of new gloves for new patients $(\mathrm{p}=0.00)$, incorrect tube collection sequences $(\mathrm{p}=0.002)$ and wrongly applying tourniquets before locating good vein $(\mathrm{p}=0.048)$, in which those taking phlebotomy training perform better than those who did not.

Table 2. Observational finding on practices after venous blood collection in public hospitals in AA, May 2014.

\begin{tabular}{lll}
\hline Observational finding & Frequency & Percentage \\
\hline Apply new gauze after blood collection & & \\
No & 200 & 100.0 \\
Yes & 0 & 00.00 \\
Recapping of needles after blood collection & & \\
No & 7 & 3.5 \\
Yes & 193 & 96.5 \\
Way of recapping needle after blood & & \\
collection & & \\
not recapped & 7 & 3.5 \\
by using one hand & 178 & 89.0 \\
by using two hand & 15 & 7.5 \\
Storing collected sample & & \\
give it to patients & 2 & 1.0 \\
laying on table & 27 & 13.5 \\
put on racks & 171 & 85.5 \\
EDTA tube blood mixing times & & \\
Acceptable (5-8x) & 122 & 61.0 \\
Unacceptable & 78 & 39.0 \\
SST tube with additives blood mixing times & & \\
Unacceptable & 157 & 78.5 \\
Acceptable & 43 & 21.5 \\
\hline
\end{tabular}

Table 3. Major Source of errors observed during phlebotomies based on phlebotomy training distributions in public hospitals in Addis Ababa, May 2014.

\begin{tabular}{|c|c|c|c|c|}
\hline \multirow{2}{*}{ Error distributions } & \multicolumn{3}{|c|}{$\begin{array}{l}\text { N (\%) Of phlebotomies } \\
\text { All (200 phlebotomies by } 40 \text { phlebotomists) }\end{array}$} & \multirow{2}{*}{ P value* } \\
\hline & $\operatorname{All}(\mathbf{n}=\mathbf{2 0 0})^{\#}$ & trained $(n=80)^{\#}$ & $\begin{array}{l}\text { untrained } \\
(\mathbf{n}=120)^{\#}\end{array}$ & \\
\hline Not using of new gloves for each new patient & $139(69.5 \%)$ & $43(53.8 \%)$ & $96(80 \%)$ & 0.000 \\
\hline Inappropriate cleaning procedure of venipuncture site & $180(90 \%)$ & $71(88.8 \%)$ & $109(91 \%)$ & 0.638 \\
\hline Collecting blood before cleaning alcohol dry & $139(69.5 \%)$ & $54(67.5 \%)$ & $85(70.8 \%)$ & 0.641 \\
\hline Incorrect tube collection sequences & $107(53.5 \%)$ & $32(40 \%)$ & $75(62.5 \%)$ & 0.002 \\
\hline Applying tourniquet before locating good sits and vain & $175(87.5 \%)$ & $65(81.2 \%)$ & $110(92 \%)$ & 0.048 \\
\hline
\end{tabular}

*chi square test used (Statistically significant i.e $\mathrm{p}<0.05$ ), \#Number of phlebotomies

Tourniquet application time was recorded for the 40 phlebotomists and is depicted in (Figure 2). The final grand mean $(\mathrm{SD})$ was 51.57 seconds $( \pm 12.514)$. Our finding indicates that appropriate tourniquet time was utilized in 148 of 200
(74\%) patients based on Clinical and Laboratory Standards Institute (CLSI) whereas 52 of $200(26 \%)$ were collected with greater than the maximum recommendation time (60 second) by CLSI recommendation. Only $2.5 \%$ collections were 
collected below 30 seconds, which is very acceptable.

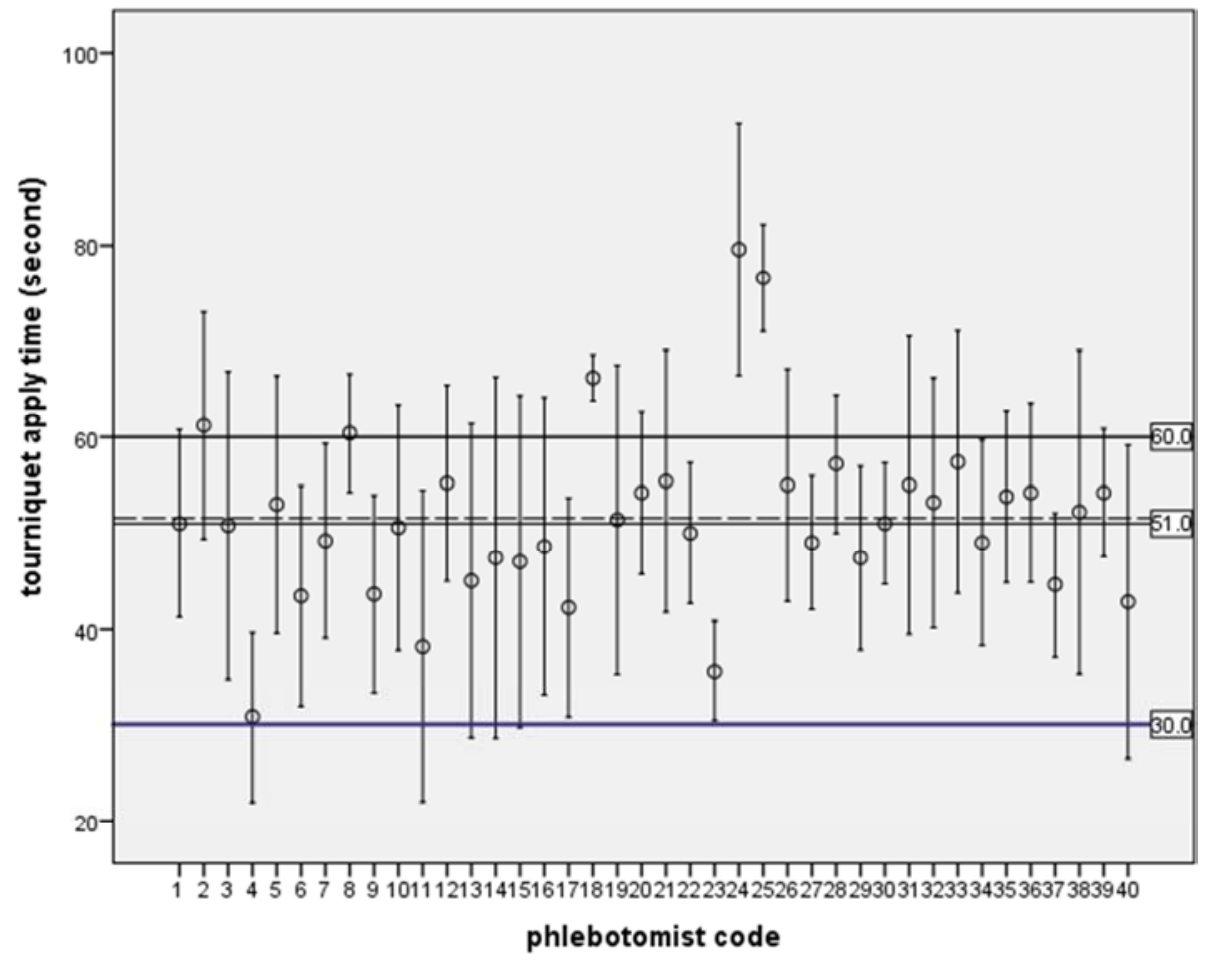

Figure 2. Tourniquet application time in seconds during phlebotomy in public hospitals in AA, May 2014, Note: Identical symbols show that the mean (SD) tourniquet time of each phlebotomist. The double line indicates that the overall mean of tourniquet application time. The solid line on 60- seconds mark shows that the maximum recommended time and the line at 30 seconds mark indicates mean time of tourniquet application time according to clinical and laboratory standards' institute (CLSI) publication H3-A5 (17).

Figure 3 showed that tourniquet application time comparison based on experience and training. From this picture we observed that there is no difference between experienced and trained for tourniquet usage, even experienced were delayed more than the mean application time but non experienced perform lower than the total mean time.

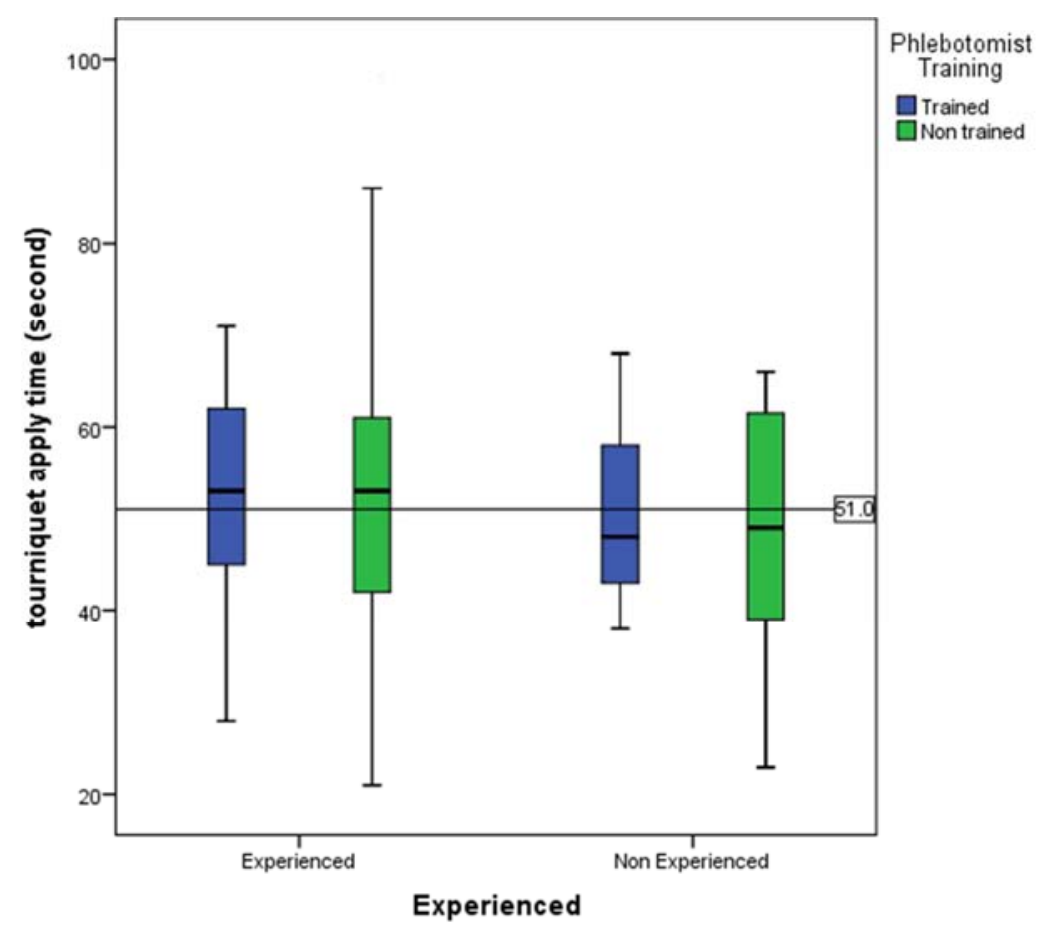

Figure 3. Tourniquet application time based on phlebotomists experience and training in public hospitals in Addis Ababa, May 2014. Note: Experienced and trained were $n=11$, experienced and non trained $n=17$, non-experienced and trained $n=5$ and non experienced and not trained $n=7$ with adjusted ratio (OR) $=0.9$. 


\section{Discussion}

Several studies have documented that most of the errors in the clinical laboratory occur in the pre analytical phase accounting up to $80 \%[2,4,6,8,9]$. Phlebotomy is most neglected but regularly practiced medical process worldwide. It can lead to adverse problems in patient safety and healthy life if not properly regulated $[1,2,4,11]$. This study aimed to assess the practice of phlebotomists and to identify the major sources of errors during venous blood collection in public hospitals in Addis Ababa, Ethiopia.

Primarily, laboratories should have clear sign and direction that will direct clients' where the laboratory sample collection area found and which prevent patients from killing their time by searching blood collection area. In this study, $80 \%$ of the phlebotomy areas were found that have clear sign that directs easily where the collection area is found and $70 \%$ had sufficient space to perform daily works and this agrees with the recommended WHO 2010 phlebotomy guideline, although $20-30 \%$ of the phlebotomy sites have to still fulfill this [1].

Safety should be implemented throughout all steps of laboratory works and Special care is required for patients during blood collections $[1,10]$ but in our study new glove were used only about $30.5 \%$ of phlebotomies, which is not recommended by WHO blood drawing guide line. According to WHO blood drawing guide line and Bowen et al [10], the puncture site should be clean properly in circular manner from the puncture site out wards slowly but in our study cleaning of collection site was performed by rubbing vigorously and irregularly; sometimes in zig zag and repeating the cleaned site with a dirty swab for $180(90 \%)$ of collections which was almost similar to the study conducted by Lima-Oliveira G et al in Brazil (85\%) [4].

WHO blood collection guideline recommends that the punctured site should be pressed to stop bleeding by using new gauze but in our study, it was found that all phlebotomists were practicing to stop bleeding by applying alcohol swabs, which were used for cleaning of punctured sites but in contrary with Program for Appropriate Technology in Health (PATH) recommendations and Lai X, et al $[1,5,14]$.

In this study Phlebotomists were requesting clients to clench unnecessarily his/her fist repeatedly for 50.5\% (101) of collections, which is incomparable with the study of LimaOliveira G, et al 2012 that reported $83 \%$ of observed patients were unnecessarily requested to clench fists repeatedly, but those practices would lead to change in the $\mathrm{PH}$ of local skin, affect electrolyte concentration like potassium, calcium and protein analyses [4].

According to PATH publications, Becton Dickinson (BD) lab notes and CLSI H3-A5 publications, sequence of blood collection with vacuum tube should be in the order of $1 \mathrm{st}$ coagulation (citrated tube), 2nd serum tube with clot activator, 3rd heparinated tube and 4th EDTA (Ethylenediaminetetraacetic acid) [8, 10, 14, 15, 17]. If this order is modified the possibility of contamination through carryover with additives will be potentially high; but, in our study, $53.5 \%$ (107 of 200) phlebotomies were collected by modifying SST with additive and EDTA tubes collection order but lower than similar problems observed $(87 \%)$ by Lima-Oliveira $\mathrm{G}$ et al 2012 findings [5].

Blood collected with additive tube should be mixed by inverting gently for effective homogeneity of additive with the blood within the tube $[14,15]$. In our study $60 \%$ of collections were not mixed which was smaller than LimaOliveira G et al 2012 findings 83\% [4].

Generally, six major sources of errors were observed in this study, which are comparable to errors identified by CLSI descriptions, BD lab notes and PATH publications. During blood collection, Phlebotomists should wear well-fitting gloves, and should also carry out hand hygiene before and after each patient procedure, before putting on gloves and after removing the gloves. But during our study 139 (69.5\%) phlebotomies were performing without changing gloves for different patients $[1,14,15,17]$.

We observed more than $80 \%$ of phlebotomists were found wrongly applying the tourniquets. Literatures indicated prolonged tourniquet application will increase the hemoconcentrations of non filterable $[1,4,10,12,14,17$, 18]. For that reason in our study time of applying of tourniquets includes with in recommended time (51.6 \pm 12.5$)$. However, this result was different with the study showed by Serdar et al as average time of less than 30 seconds [12]; but, our finding disproof the finding of Lina-Oliver $\mathrm{G}$, et al that the overall mean (SD) application time of $84.4 \pm 14.1$ [4].

\section{Conclusion and Recommendations}

\subsection{Conclusion}

According to our study findings the following conclusions were drawn;

Most phlebotomy areas were having a clear sign of location, clean patients waiting area and requests contains space to record information of the patients, have no SOPs that directs how to collect, process and handle laboratory specimens but few have job aids

Most of the phlebotomy area were not well ventilated and could be the cause of infection for phlebotomist and patients

The Majority of the phlebotomist was not getting phlebotomy training and even those who have training and who responded as they have enough knowledge on phlebotomy service were not well practicing phlebotomy.

The main errors were using of a single gloves for different patient, vigorously and inappropriately cleaning of vein collection site, collection of blood specimens before disinfectant alcohol dry, incorrectly using of collection tube sequence, collecting with syringe and transferring to vacutainer tubes some even by opening the tubes with additives, applying of tourniquets even after blood flow started into the tube or syringe and tourniquet application 
before examination of appropriate collection site

\subsection{Recommendations}

Based on the finding of the result about the phlebotomy service and phlebotomy practice the following recommendations are forwarded:

1. Laboratories should have clear sign and direction that will direct clients' where the laboratory sample collection is found (some in this study does not have signs).

2. No specimen collection SOPs found in almost all phlebotomy area; so general phlebotomy SOPs should be provided and available in collection areas. The situation might even be worse in patient venous blood collections, thus, SOPs should be available with appropriate training in all collection sites.

3. Laboratory managers, supervisors or quality officers at each facility should take responsibility to ensure quality of laboratory specimen

4. Phlebotomists are performing their daily work with infectious and the possibility of injury conditions so phlebotomists should be vaccinated.

\section{Limitations of the Study}

Interviewed results were dependent on Phlebotomist response so biased information might be given as data were collected during their daily working time. However, the observational data help to minimize this bias.

The study covers only phlebotomists that are found during the study period, so those who are not in working site during the study time were not included and the study only observe vein blood collection practice, we have not included other specimen collection and all specimen handling up to result delivering.

\section{Competing Interests}

We, the authors declare that, we have no financial or personal relationship(s) that may have inappropriately influenced us in writing this work

\section{Authors' Contributions}

Wondimeneh Liknew conceived and designed the study and collected data, performed analysis, interpretation of data. Abay Sisay, Dr. Aster Tsegaye, Eshetu Lema had assisted with the design, performed, analysis, interpretation of data, drafted and critically reviewed this manuscript.

\section{Acknowledgment}

First of all we would like to thank to our almighty GOD who endowing us good health throughout our life. We grateful to Addis Ababa University, School of clinical Laboratory Science, Addis Ababa City Government Health Bureau for granting approval. Our thanks also extend to those all who cooperated with us in doing this work, especially laboratory professionals, data collectors, supervisor and the study subjects who participated in one way or another on this study.

\section{References}

[1] WHO Guidelines on Drawing Blood: Best Practices in Phlebotomy. Geneva; 2010.

[2] Lippi G, Salvagno GL, Montagnana M, Franchini M, Guidi GC. Phlebotomy issues and quality improvement in results of laboratory testing. Clinical laboratory. 2006; 52 (5-6): 217-230.

[3] Hawkins R. Managing the pre-and post-analytical phases of the total testing process. Ann Lab Med. 2012; 32 (1): 5-16.

[4] Lima-Oliveira G, Guidi GC, Salvagno GL, Montagnana M, Rego FG, Lippi G, et al. Is Phlebotomy Part of the Dark Side in the Clinical Laboratory Struggle for Quality? Lab Med. 2012; 43 (5): 172-176.

[5] Lai X, Yang P, Zhang Y, Cao J, Zhang L. Analysis of Factors Influencing the Generation of Unqualified Clinical Samples and Measures to Prevent this Generation. Ann Lab Med. 2012; 32 (3): 216-219.

[6] Mäkitalo O, Liikanen E. Improving Quality at the Preanalytical Phase of Blood Sampling: Literature Review. International Journal of Biomedical Laboratory Science (JIBS). 2013; 2 (1): 7-16.

[7] Bolenius K, Brulin C, Grankvist K, Lindkvist M, Soderberg J. A content validated questionnaire for assessment of self reported venous blood sampling practices. BMC Res Notes. 2012; 5: 39. 1

[8] Saurav Patra M, Mukherjee B, Das AK. Pre-analytical errors in the clinical laboratory and how to minimize them. Int. J. Bioassays. 2013; 2 (3): 551-553.

[9] Söderberg J, Wallin O, Grankvist K, Brulin C. Is the test result correct? A questionnaire study of blood collection practices in primary health care. J Eval Clin Pract. 2010; 16 (4): 707-711.

[10] Bowen RA, Hortin GL, Csako G, Otanez OH, Remaley AT. Impact of blood collection devices on clinical chemistry assays. Clin Biochem. 2010; 43 (1-2): 4-25.

[11] Lippi G, Becan-McBride K, Behúlová D, Bowen RA, Church $\mathrm{S}$, Delanghe J, et al. Preanalytical quality improvement: in quality we trust. Clin Chem Lab Med. 2013; 51 (1): 229-241.

[12] Serdar MA, Kenar L, Hasimi A, Kocu L, Turkmen YH, Kurt I, et al. Tourniquet application time during phlebotomy and the influence on clinical chemistry testing; is it negligible. Turk J Biochem. 2008; 33: 85-88.

[13] Abay Sisay, Tedla Mindaye, Abrham Tesfaye, Eyob Abera, Adino Desale, Assessing the outcome of Strengthening Laboratory Management Towards Accreditation (SLMTA) on laboratory quality management system in city government of Addis Ababa, Ethiopia. The pan African Medical Journal. 2015; 20: 314 .

[14] Program for Appropriate Technology in Health (PATH). RBPEIA: Collecting, Processing, and Handling Venous, Capillary, and Blood Spot Samples. PATH Publications. 2005. [http://www.path.org (accsessed date 16 september 2013)]. 
[15] Bush V, Mangan L. The Hemolyzed Specimen: Causes, Effects, and Reduction. BD Lab Notes. 003; 13 (1). [http://www.bd.com/vacutainer (accessed date 12 september 2013)].

[16] Sultan J, Afzal T, Jabeen K. Identification of the Types of Preanalytical Errors in the Clinical Chemistry Laboratory from Jan-2012 to Dec-2012 at Jinnah Hospital, Pathology Department. A. P. M. C. 2013; 7:1 (accsessed date 1 september 2013)].
[17] Clinical and Laboratory Standards Institute (CLSI). Procedures for the collection of diagnostic blood specimens by venipuncture. Document H3-A5. 2003; 23 (32): 17.

[18] Lippi G, Salvagno GL, Montagnana M, Brocco G, Guidi GC. Influence of short-term venous stasis on clinical chemistry testing. Clin Chem Lab Med. 2005; 43 (8): 869-75. 